\title{
A Web-Based Virtual Experiment in Material Science: Tensile Test Laboratory Application
}

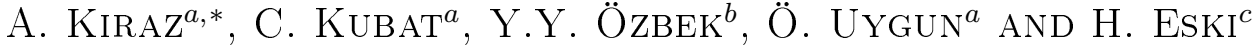 \\ ${ }^{a}$ Department of Industrial Engineering, Faculty of Engineering, Sakarya University \\ Sakarya University Esentepe Campus, 54187 Serdivan, Sakarya, Turkey \\ ${ }^{b}$ Department of Metallurgical and Materials Engineering, Faculty of Engineering, Sakarya University, Turkey \\ ${ }^{c}$ Department of Computer Engineering, Faculty of Computer and Informatics, Sakarya University, Turkey \\ Virtual laboratories have a very important place for distant education which has real applications of labora- \\ tory tests and experiment applications. It is fact that virtual laboratories necessity is inevitable because of the \\ requirements of materials, place, staff and above all, time and financial requirements for establishment of real \\ laboratories. The main concept of virtual laboratory is to replace real machines with their virtual simulations. \\ Real investigative equipment is not often available for students, researchers, and practitioners as users in addition \\ to their expensive costs in usage. Virtual laboratories are cheap and safe in use because all mistakes can be easily \\ erased by web-based application or simulation reset without any consequences. Furthermore users can easily make \\ many tests regardless of place in any time on the web. In this study AISI 4140 steel was used, since AISI 4140 \\ steel is the most well-known type of steel used in industries. Tensile test of the steel was examined regarding to \\ different tensile speeds. The study aimed to design the web-based virtual tensile test laboratory. The uniqueness \\ of this study is generating an artificial neural network model by using the values of the material which is stressed \\ in different speeds. Thanks to this model, intermediate speed values were predicted. Besides that, this model was \\ used to design web-based virtual tensile test laboratory application. With the help of this application, users can \\ easily realize the yield strength, ultimate strength and fracture strength on stress-strain diagram.
}

DOI: 10.12693 /APhysPolA.125.310

PACS: 07.10.Lw

\section{Introduction}

Engineering education has a big importance in development of technologies. For this purpose, various methods have been developed to improve learning processes. The technological researches and developments provide new concepts in education [1]. Especially internet facilities present new approaches and methods to design virtual and real time laboratory applications for education purposes. Laboratory work is an important part of the engineering course, in which the students can make practice of what they learned in the classes, which helps them to reinforce the learning of the concepts [2].

Virtual laboratories (VL) necessity is inevitable because of the requirements of materials, place, staff and above all, time and financial requirements for establishment of real laboratories [3]. Facilities of the laboratories in educational institutions are generally insufficient when the number of students is considered. Implementation of a laboratory to meet the requirements has a very high price.

The internet provides a new environment for developing a variety of applications for educational and research purposes. This paper presents the implementation of a web-based laboratory experiment on a tensile

*corresponding author; e-mail: kiraz@sakarya.edu.tr test. The web-based laboratory has been developed to serve students and staff in the Department of Metallurgical and Materials Engineering at the Sakarya University of Turkey. The laboratory is an educational tool for teaching students the basic principles and methodology in performing a series of experiments on a tensile test in different speeds at any time and from any location through the internet. With the help of this application users can easily observe the difference in the stress-strain diagram if tensile speed is changed.

In this study tensile test was examined for virtual laboratories and it is also aimed at designing the tensile test in virtual environments for different tensile speeds. An artificial neural network was generated from the values of a material which was stressed in different speeds. Intermediate speed values were predicted by this artificial neural network. This artificial intelligence technique is implemented to web and thanks to this application, users can change the tensile speed to see the changes on stress-strain diagram and also yield strength, ultimate strength, and fracture strength.

\section{Tensile test}

Tensile testing is one of the most fundamental tests for engineering, and provides valuable information about a material and its associated properties. These properties can be used for design and analysis of engineering structures, and for developing new materials that better suit a specified use. Tensile test is a mechanical test 
where a pulling force is applied to a material from both sides until the sample changes its shape or breaks. It is a common and important test that provides a variety of information about the material being tested, including the elongation, yield point, tensile strength, and ultimate strength of the material. These tests are commonly performed on substances such as metals, plastics, wood, and ceramics.

The most common type of test used to measure the mechanical properties of a material is the tensile test. Tensile test is widely used to provide basic design information on the strength of materials and is an acceptance test for the specification of materials. The major parameters that describe the stress-strain curve obtained during the tension test are the tensile strength (UTS), yield strength or yield point $(\sigma y)$, elastic modulus $(E)$, percent elongation $(\Delta L \%)$ and the reduction in area (RA\%). Toughness, resilience, Poisson's ratio $(\nu)$ can also be found by the use of this testing technique [4].

In this test, a specimen shown in Fig. 1 is prepared suitably for gripping into the jaws of the testing machine type that is used.

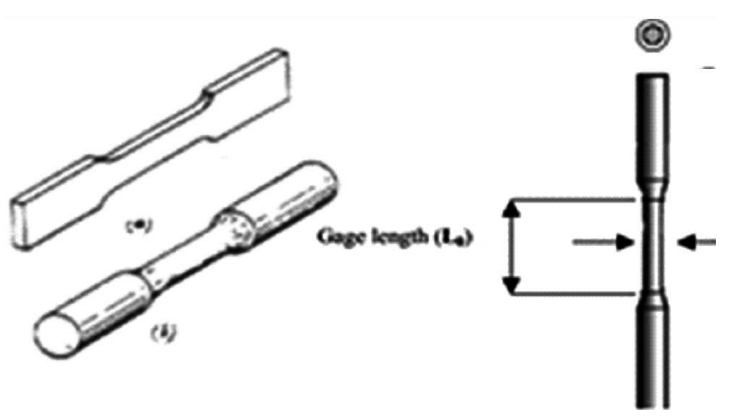

Fig. 1. Tensile specimens (a) rectangular, round [4].

In the study, realization of effect of tensile speed to the stress-strain diagram in virtual environment with artificial neural network is aimed. For this reason, the same sample groups are stressed in different speeds in tensile test to observe changes in stress-strain diagram. Materials' tensile tests are made 3 times in each speed with different speed value as $4,8,10,12,14,16,18$, and $20 \mathrm{~mm} / \mathrm{min}$.

\section{Virtual tensile test application}

Formerly laboratory applications were used to prove theoretical knowledge but lately they turned into environments where students freely discover knowledge as an individual or in groups. The activities that have come up with the recent form of labs substantially contributed to training ideal students for constructivist approach, who research, inquire, test, seek solutions, and deeply reason about the concept of concern. However, on the present stage of our educational system, these activities cannot be included in lessons for several reasons. At that point virtual labs emerged as an alternative solution for the problems of the instruction of applied courses.

The main negative factors of the current laboratories are shown below.

- In carrying out experiments and arranging with equipment, the laboratory activities are expensive.

- For planning and application, it is much time consuming.

- Checking students' performance during the activities can be difficult in over-crowded classes.

- Lack of laboratory or equipment, or insufficient lab conditions which limits the teacher to perform a simple lab activity [5].

We can clearly see from these factors that virtual laboratories necessity is inevitable. Students, researchers and practitioners overcome the possible dangers that can be seen in the real laboratory conditions. For example a dangerous experiment for human health is prepared in computer as simulations, so that students can see the experiments design and perform the experiment in computer and observe the result. Other than performing dangerous, difficult or impossible experiments, simulations have advantages from the time, security, cost and motivation point of view $[6,7]$. The main objectives of virtual laboratories are shown below.

- To provide remote-access to labs in various disciplines of science and engineering. These virtual labs would cater to students at the undergraduate level, post graduate level, as well as to research scholars.

- To enthuse students to conduct experiments by arousing their curiosity. This would help them in learning basic and advanced concepts through remote experimentation.

- To provide a complete learning management system around the virtual labs where the students can avail the various tools for learning, including additional web-resources, video-lectures, animated demonstrations and self-evaluation.

- To share costly equipment and resources, which are otherwise available to limited number of users due to constraints on time and geographical distances [8].

Application was designed web-based to improve its accessibility for practitioners. In this web-based application, HTML5 was used for creating graphics and designing interface. MySQL database was used for warehousing test data, users' information, and others. An artificial neural network model was created by using Matlab to predict intermediate tensile speed values. This model was implemented to PHP script language for predict process and user interaction. Figure 2 shows web-based tensile test application. 


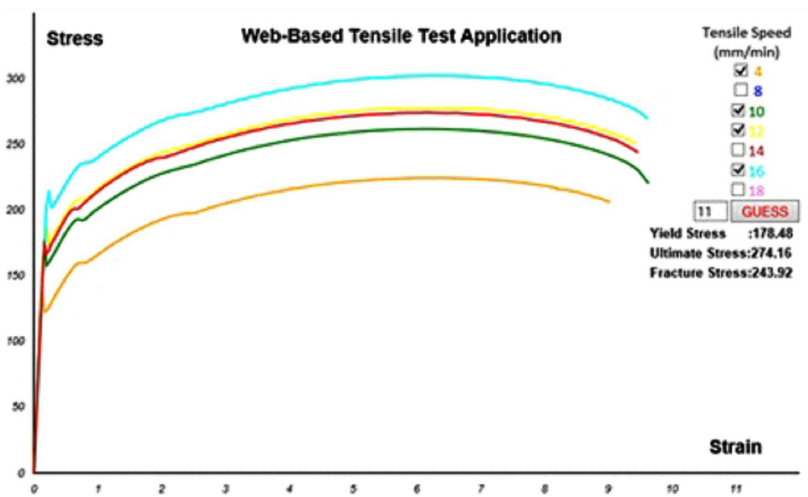

Fig. 2. Web-based tensile test application.

\section{Conclusions}

Virtual laboratory applications can be used as a supportive tool in real labs or as an alternative lab where there is no available physical laboratory or conditions of the physical lab are insufficient. Literature review shows that students in the sample group state that they prefer conducting experiment in virtual laboratory to real labs and they think that it is easier and more understandable to do experiments with the help of software's. Virtual laboratory software's create very effective learning environment, support constructivist approach and students centered education, promise great performance with low cost. It is observed that VL software positively affects the success attitudes, and motivation of the students and it enables the student to recognize the learnt concepts more easily. It is also mentioned that VL is an alternative to a real lab when the experiments cannot be conducted in a real lab for some reasons.
In this study, we have studied the factor, deformation rate, affecting the stress-strain diagram. The different deformation rates $(4,8,10,12,14,16,18 \mathrm{~mm} / \mathrm{min})$ were used for training data of artificial neural network model. This model implemented to web-base and $11 \mathrm{~mm} / \mathrm{min}$ strain-stress diagram was predicted. Users can change the deformation rate to see the changes on the diagram. They also can see the values of yield strength, ultimate strength and fracture strength.

\section{Acknowledgments}

This work is supported by Sakarya University Scientific Research Foundation (project No. 2012-01-02-006).

\section{References}

[1] H.A. Basher, S.A. Isa, Proc. IEEE SoutheastCon., 325 (2006).

[2] M. Jurèeviæ, M. Malariæ, A. Sala, Measur. Sci. Rev. 6, 36 (2006)

[3] C. Kubat, A. Kiraz, J. Faculty Eng. Architect. Gazi University 27, 205 (2012).

[4] R. Gürbüz, G. Cevik, Lecture Note, Middle East Technical University, Department of Metallurgical and Materials Engineering, Ankara 2010, p. 1.

[5] C. Tüysüz, Int. Online J. Educat. Sci. 2, 37 (2010).

[6] S. Rodrigues, Austral. Sci. Teachers J. 43, 38 (1997).

[7] M. Tekdal, Development and Effective Usage of Interactive Physics Simulations in: $V$ National Congress on Science and Mathematics Education, ODTÜ, Ankara 2002 (in Turkish).

[8] http://www.vlab.co.in/ (Access Date: 20.07.2013). 